\title{
La fécondation par injection d'une spermatide dans l'ovule
}

\author{
J. TESTART
}

INSERM, Unité 355 "Maturation gamétique et fécondation" 32, rue des Carnets, 92140 Clamart ; Laboratoire d'AMP, Hôpital Américain, 63 Boulevard Victor Hugo, 92202 Neuilly

\section{RESUME}

La technique de fécondation in vitro (FIV) par injection intracytoplasmique d'un spermatozoïde (ICSI) s'est rapidement répandue dans les centres de FIV. Les résultats récents confirment non seulement l'efficacité de la technique, mais aussi son innocuité, les rares anomalies observées chez les enfants pouvant être imputées à l'héritage du génome paternel. L'ICSI rend inutiles les fonctions acquises par le gamète issu de la méiose (spermatide) quand il subit la spermiogenèse pour se transformer en spermatozoïde. Ces fonctions (mobilité, protection du génome, systèmes de reconnaissance et de pénétration de l'ovocyte) peuvent donc être considérées comme le tribut payé par la spermatogenèse pour rendre possible la fécondation dans l'organisme féminin, chez tous les mammifères. L'hypothèse d'une "surmaturité" du gamète masculin, rendue nécessaire par les aléas de la fécondation naturelle, est confortée par la comparaison des gamétogenèses mâle et femelle puisque l'ovocyte "mûr" est méiotiquement plus immature que la spermatide.

A l'issue d'expériences réussies chez le lapin et la souris, des spermatides ont été à l'origine de plusieurs naissances normales après ICSI dans l'espèce humaine (France, Angleterre, Belgique). Dans tous les cas les hommes étaient azoospermiques et les spermatides ont été recueillies soit dans le sperme éjaculé, soit dans le testicule. La place de l'ICSI avec spermatides dans l'AMP dépend de plusieurs facteurs encore mal connus actuellement. Nous avons évalué à $76 \%$ la proportion d'hommes souffrant d'azoospermie non obstructive qui présentent des spermatides dans leur éjaculat. L'ICSI avec spermatides pourrait donc concerner 5 à $10 \%$ des hommes stériles.

Mots clés : spermatide, ICSI, méiose, fécondation.

Si la stérilité féminine peut être palliée avec un certain succès grâce à la FIV (environ $20 \%$ de grossesses cliniques par ponction d'ovocytes) les altérations du sperme constituent un handicap considérable qu'on tentait jusqu'à récemment de résoudre par l'insémination artificielle ou la FIV, en ayant recours à un don de sperme dans les cas les plus défavorables (Tableau 1 ). Cependant, il existe des situations extrêmes ou même la FIV est impuissante, le nombre de gamètes mobiles disponibles étant très insuffisant alors que le couple stérile n'accepte pas l'éventualité d'un tiers donneur. 
Tableau 1 : Grossesses pour 100 cycles de traitement selon la technique d'AMP et la qualité du sperme.

\begin{tabular}{lcccccc}
\hline Fertilité de l'homme & NORMALE & \multicolumn{2}{c}{ REDUITE } & \multicolumn{2}{c}{ NULLE } \\
\hline Technique d'AMP & FIV-C & IAC & FIV-C & IAD & FIV-D ICSI \\
$\%$ grossesses & 20 & $5-20$ & 16 & 9 & 23 & 23 \\
\hline
\end{tabular}

Tableau 2 : Nombre de spermatozoüdes pour féconder un ovocyte.

\begin{tabular}{lcr}
\hline $\begin{array}{l}\text { Méthode de } \\
\text { fécondation }\end{array}$ & niveau & nombre de \\
& d'insémination & spermatozoïdes
\end{tabular}

- rapport sexuel vagin 200000000

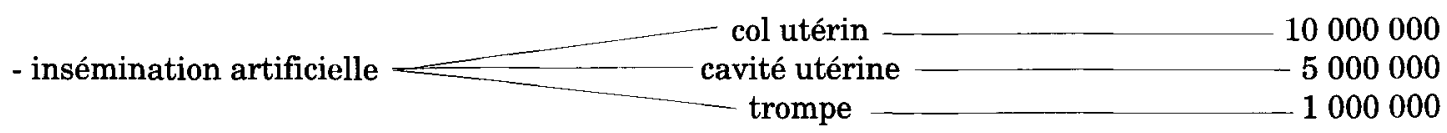

- fécondation in vitro

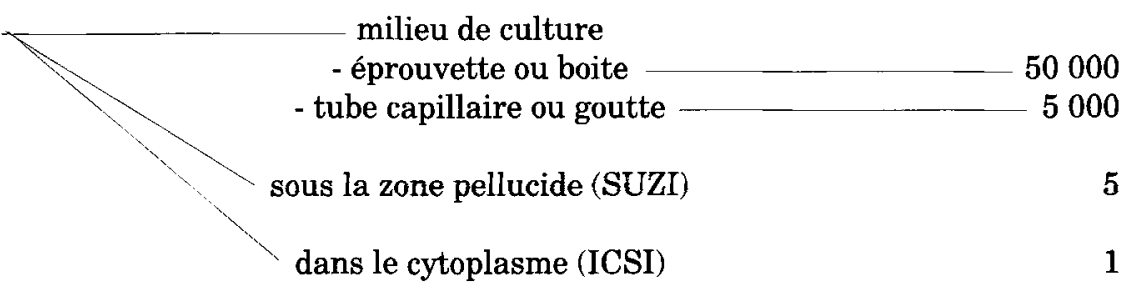

C'est pourquoi, depuis la première démonstration de fécondation par injection du spermatozoïde dans le cytoplasme ovulaire [21] la technique d'ICSI a pris une place très importante dans la plupart des Centres d'AMP. Les dernières statistiques du groupe pionnier [3] indiquent que les résultats de l'ICSI sont équivalents à ceux de la FIV pratiquée avec un sperme normal et que les enfants nés après ICSI ne montrent pas d'anomalie particulière, sauf une augmentation $(\sim 1 \%)$ de la fréquence des aneuploïdies concernant les chromosomes sexuels. Ce phénomène est généralement attribué à la transmission d'une anomalie paternelle, telle une mosaïque [25], mais il n'a cependant pas été retrouvé dans une récente enquête des biologistes français (BLEFCO, 2) = Parmi 611 enfants nés après ICSI on ne recensait que 2 syndromes de Klinefelter et 1 syndrome de Turner $(0,5 \%)$.

Actuellement, environ une tentative de FIV sur quatre est réalisée par ICSI en France selon FIVNAT [8] bien que les indications légitimes paraissent respectées [2]. Ainsi le nombre de spermatozoïdes nécessaires pour féconder un ovocyte a rapidement diminué de 200 millions à l'unité (Tableau 2), tandis que le nombre d'ovocytes disponibles décuplait, grâce aux traitements de stimulation ovarienne.

\section{L'ICSI AVEC GAMETE ATYPIQUE}

Lors de l'ICSI, il est convenu de recourir à l'injection des spermatozoïdes les plus typiques (mobiles et de forme normale) 
chaque fois que possible, afin de mimer la situation naturelle. Pourtant des circonstances expérimentales ou cliniques ont permis d'explorer la fécondance de gamètes atypiques, et d'évaluer la qualité de l'oeuf ainsi obtenu. Chez la souris, la sélection volontaire de spermatozoïdes morphologiquement très anormaux (tête très déformée) n'empêche pas la procréation par ICSI [5]. Ces résultats indiquent que le gamète anormal contient le génome et les organites compatibles avec le développement de descendants fertiles; de plus, les auteurs [5] observent un sperme amélioré (spermatozoïdes plus typiques) chez les descendants, par comparaison avec les gamètes paternels, soulignant ainsi la dissociation entre génotype et phénotype du spermatozoïde. D'autres travaux montrent que la tête isolée du spermatozoïde de souris, maltraitée de diverses façons (sonication, triton, démembranation, congélation brutale ...) reste compétente pour produire des souriceaux normaux par ICSI [13]. Ces résultats montrent que, chez la souris, la fécondation viable ne nécessite aucun des organites extra nucléaires pourvu que l'activation de l'ovocyte soit artificiellement induite. Citons aussi l'ICSI, dans l'ovocyte de souris, de spermatozoïdes humains dépourvus d'acrosome [24], expérience qui montre que l'anomalie spermatique ne correspond pas à une anomalie chromosomique. C'est encore l'équipe de Yanagimachi qui a récemment montré que la sélection volontaire de spermatozoïdes humains anormaux n'a pas d'influence notable sur les anomalies résultant de l'ICSI dans l'ovocyte de souris [14] :malgré une fréquence augmentée d'anomalies structurelles quand le gamète mâle présente une tête très déformée, ou ronde, ou allongée, le taux d'aneuploïdie est normal. Le même travail (14) ne relève pas d'effet sur les anomalies chromosomiques quand le spermatozoïde est microcéphale ou macrocéphale.

Ainsi se trouve confirmé dans l'espèce humaine l'absence de relation stricte entre l'aspect du spermatozoïde et sa constitution chromosomique. Par ailleurs, cette équipe a aussi eu recours à l'ICSI pour rendre fertiles des souris stériles parce que leur génome s'oppose à l'interaction gamétique [12]. Cette expérience constitue un modèle animal pour d'éventuelles interventions permettant de pallier une stérilité humaine d'origine génétique telle que des microdélétions du bras long du chromosome Y [23] ou la non fonctionnalité du gène $\operatorname{CREM}[4,17]$ par exemple. Dans ces cas une descendance complètement normale, sauf pour sa fertilité, peut être obtenue après ICSI.

Dans l'espèce humaine aussi, la fécondation avec des spermatozoïdes anormaux a été réalisée dans le cadre d'AMP comme l'insémination subzonale (SUZI) de gamètes immobiles par absence de bras externes de dynéine [38]. De plus, en cas d'azoospermie, l'ICSI est couramment pratiquée avec des spermatozoïdes obtenus par ponction de l'épididyme ou du testicule [26]. Ainsi la totalité des événements en aval de la production des spermatozoïdes (maturation épididymaire, capacitation, reconnaissance gamétique, réaction acrosomique, fusion membranaire) peut être court-circuitée sans conséquence pour la procréation. La grossesse a même été obtenue par ICSI de spermatozoïdes testiculaires dont l'immobilité persistait malgré l'incubation in vitro [18], soulignant que les défauts cellulaires responsables de l'immobilité n'annulent pas la capacité nucléaire à permettre une fécondation normale. Quant à la morphologie des spermatozoïdes humains, pas plus que chez l'animal elle n'a pu être mise en relation avec les résultats de l'ICSI, lesquels sont indépendants du spermocytogramme [32].

Ainsi la seule exigence pour la fécondation par ICSI est la présence d'un spermatozoïde vivant [16] quelles que soient ses caractéristiques et son origine. Si une étude cytologique des événements de la fécondation par ICSI chez le singe Rhésus [31] révèle certaines différences par rapport à 
la fécondation naturelle, rien n'indique que ces différences puissent avoir d'éventuelles conséquences sur le développement.

\section{L'ICSI AVEC GAMETE IMMATURE}

En amont de l'immaturité physiologique qui affecte le spermatozoïde testiculaire, des gamètes n'ayant pas subi la totalité de la spermatogenèse ont été injectés dans des ovules dans le but d'obtenir la fécondation et le développement. Comme l'indique le Tableau 3, l'ICSI avec spermatide a été réalisée dans plusieurs espèces animales (hamster, souris, lapin, bovin), et quelques dizaines de nouveaux nés normaux, capables de procréer, ont été obtenus chez la souris et le lapin. On note qu'il s'agissait de spermatides rondes, c'est-à-dire en tout début de spermiogenèse, et que le plus souvent le noyau de ces cellules seulement était injecté, d'où la nécessité de procéder à l'activation artificielle de l'ovocyte, le facteur spermatique cytosolique ou "oscilline» [22] étant absent. De plus, ces spermatides étaient recueillies dans le testicule plutôt que dans la semence (réalisable seulement chez le lapin et le taureau) ou dans l'épididyme (origine usuelle des spermatozoïdes utilisés en FIV chez les rongeurs). Bien qu'aucune allusion à ce choix ne figure dans les articles cités, on admettra que chez ces mâles normalement fertiles c'est dans le testicule qu'il est logique de rechercher des spermatides.

Tableau 3 : Fécondation avec précurseurs du spermatozoïde.

\begin{tabular}{|c|c|c|c|c|c|c|}
\hline Espèces & Origine & Stade & Etat & Technique & Résultats & Références \\
\hline Hamster & testicule & $\begin{array}{l}\text { spermatide } \\
\text { ronde }\end{array}$ & noyau & ICSI & fécondation & $\begin{array}{l}\text { Ogura et } \\
\text { Yanagimachi [19] }\end{array}$ \\
\hline \multirow[t]{3}{*}{ Souris } & testicule & $\begin{array}{l}\text { spermatide } \\
\text { ronde }\end{array}$ & intacte & électrofusion & 4 petits & $\begin{array}{l}\text { Ogura, Matsuda et } \\
\text { Yanagimachi }[20]\end{array}$ \\
\hline & testicule & $\begin{array}{l}\text { spermatide } \\
\text { ronde }\end{array}$ & noyau & ICSI & 37 petits & $\begin{array}{l}\text { Kimura et } \\
\text { Yanagimachi [10] }\end{array}$ \\
\hline & testicule & spermatocyte II & noyau & ICSI & 7 petits & $\begin{array}{l}\text { Kimura et } \\
\text { Yanagimachi [11] }\end{array}$ \\
\hline \multirow[t]{2}{*}{ Lapin } & testicule & $\begin{array}{l}\text { spermatide } \\
\text { ronde }\end{array}$ & noyau & ICSI & 3 petits & Sofikitis et al. [28] \\
\hline & testicule & $\begin{array}{l}\text { spermatide } \\
\text { ronde }\end{array}$ & noyau & ICSI & 14 petits & Sofikitis et al. [30] \\
\hline Bovin & testicule & $\begin{array}{l}\text { spermatide } \\
\text { ronde }\end{array}$ & entière & ICSI & blastocystes & Goto et al. [9] \\
\hline \multirow[t]{4}{*}{ Humain } & testicule & $\begin{array}{l}\text { spermatide } \\
\text { ronde }\end{array}$ & noyau & ICSI & $4 \mathrm{FCS}$ & Sofikitis et al. [29] \\
\hline & testicule & $\begin{array}{l}\text { spermatide } \\
\text { allongée }\end{array}$ & intacte & ICSI & fécondation & $\begin{array}{l}\text { Vanderzwalmen et } \\
\text { al. [36] }\end{array}$ \\
\hline & testicule & $\begin{array}{l}\text { spermatide } \\
\text { ronde }\end{array}$ & intacte & ICSI & 1 grossesse & Fishel et al. [7] \\
\hline & éjaculat & $\begin{array}{l}\text { spermatide } \\
\text { ronde }\end{array}$ & intacte & ICSI & 2 naissances & Tesarik et al. [33] \\
\hline
\end{tabular}


Dans l'espèce humaine, Vanderzwalmen et al. obtenaient la fécondation par ICSI de spermatides allongées en 1995 [36], tandis que Sofikitis et al faisaient connaître succinctement [29] plusieurs fausses couches spontanées survenues dans des grossesses obtenues par ICSI de noyaux de spermatides rondes. Peu après des grossesses évolutives étaient rapportées simultanément par Fishel et al (une fille normale [7]) et Tesarik et al (deux garçons normaux [33]).

\section{LE SPERMATOZOÏDE EST UNE SPERMATIDE SPECIALISEE POUR LA FECONDATION}

Nous avons développé ailleurs [34] l'hypothèse que la transformation de la spermatide en spermatozoïde est le tribut payé par la spermatogenèse aux nécessités de la fécondation dans l'organisme féminin. Car il faut bien que le gamète mâle se déplace (rôle du flagelle et de la compaction nucléaire), qu'il reconnaisse l'ovule (rôle des protéines de surface) et soit capable de le pénétrer (rôle des enzymes acrosomiques). Aussi, malgré son aspect spectaculaire sur le plan cytologique, la spermiogenèse est une métamorphose cellulaire dont la signification est exclusivement fonctionnelle. Cette hypothèse, basée sur les connaissances scientifiques, est confortée par les résultats déjà obtenus grâce à l'ICSI avec spermatides chez les mammiferes, et s'oppose à diverses spéculations. Parmi cellesci, le rôle postulé de certains organites du gamète dans le développement embryonnaire qui serait empêché, ou encore l'empreinte génomique qui ne serait pas normalement réalisée. S’il est exact que, au contraire de la souris, c'est le spermatozoïde qui apporte le centriole dans l'oeuf humain, cet organite préexiste dans la spermatide et est introduit dans l'ovule par l'ICSI quand la spermatide entière est injectée. Quant à l'empreinte génomique, elle prend place avant la méiose [37], et est donc acquise dans la spermatide; certaines méthylations géniques surviennent dans l'épididyme [1] mais, si elles étaient indispensables pour un développement normal, la fécondation avec des spermatozoïdes testiculaires serait tout aussi problématique.

C'est surtout l'idée reçue que l'ovule est un gamète "mûr" tandis que la spermatide serait une cellule germinale "immature" qui vient dramatiser le débat autour de l'ICSI avec spermatide. Rappelons que la correspondance méiotique doit associer spermatocyte II avec ovocyte II c'est-à-dire avec l'ovule. Rappelons aussi que la spermatide est une cellule $n$ haploïde tandis que l'ovule est $2 \mathrm{n}$ haploïde puisque les chromatides y sont encore appariés. La belle expérience de Kimura et Yanagimachi [11], chez la souris, démontre que la fécondation normale est obtenue par ICSI du spermatocyte II dans son homologue, l'ovule. Dans ce cas chaque cellule méiotique émet son propre globule polaire. Cette démonstration soutient notre hypothèse que, du point de vue génétique, le gamète mûr est bien la spermatide, et que sa transformation en spermatozoïde correspond seulement aux nécessités de la fécondation. Notons enfin que, même si la fécondation avec spermatocyte II était réalisable dans notre espèce, il n'est pas certain que cet artifice extrême correspondrait à des indications de stérilité masculine.

\section{L'ICSI AVEC SPERMATIDE : POUR QUI ?}

Par principe, le recours à des spermatides pour l'ICSI ne concernerait que des hommes chez lesquels aucun spermatozoïde vivant n'a été recueilli dans l'éjaculat ou dans le testicule. L'évaluation de la proportion des azoospermies non obstructives pouvant bénéficier de l'ICSI avec spermatide dépend donc de la stratégie médicale envers les patients azoospermiques chez lesquels pourraient persister des foyers isolés de spermiogenèse. Bien que de tels foyers aient été trouvés chez 
certains hommes azoospermiques [27] leur recherche est tout à la fois aléatoire et iatrogène. Aussi, la décision logique de préférer injecter un spermatozoïde plutôt qu'une spermatide n'est pas nécessairement conforme à l'intérêt du couple : la répétition de biopsies testiculaires risque ne pas déboucher sur le recueil de gamètes mûrs, la totalité des tubules séminifères ne pouvant être examinée, tandis que cette intervention invasive risque de compromettre l'accès ultérieur à une source résiduelle de gamètes, même immatures. En effet, les deux causes les plus fréquentes de l'azoospermie non obstructive, les syndromes de "Sertoli cell only" et d'“arrêt de maturation" sont justement définies par l'absence de gamètes dans les biopsies testiculaires. Il a été récemment montré que les testicules de la moitié des hommes présentant les syndromes "Sertoli cell only" [27] ou d"'arrêt de maturation" [35] recèlent des foyers de tubes séminifêres normaux où se déroule la spermatogenèse. Mais un tel bilan nécessite que "the entire testis is carefully sampled" selon Silber et al [27] ... Même si, chez la souris, l'ICSI de spermatides est moins efficace que l'ICSI de spermatozoïdes [10], et en admettant que ce handicap ne soit pas résolu par des améliorations techniques, la question se pose de savoir s'il faut s'acharner à découvrir d'éventuels spermatozoïdes dans les testicules d'hommes azoospermiques mais excrétant des spermatides.

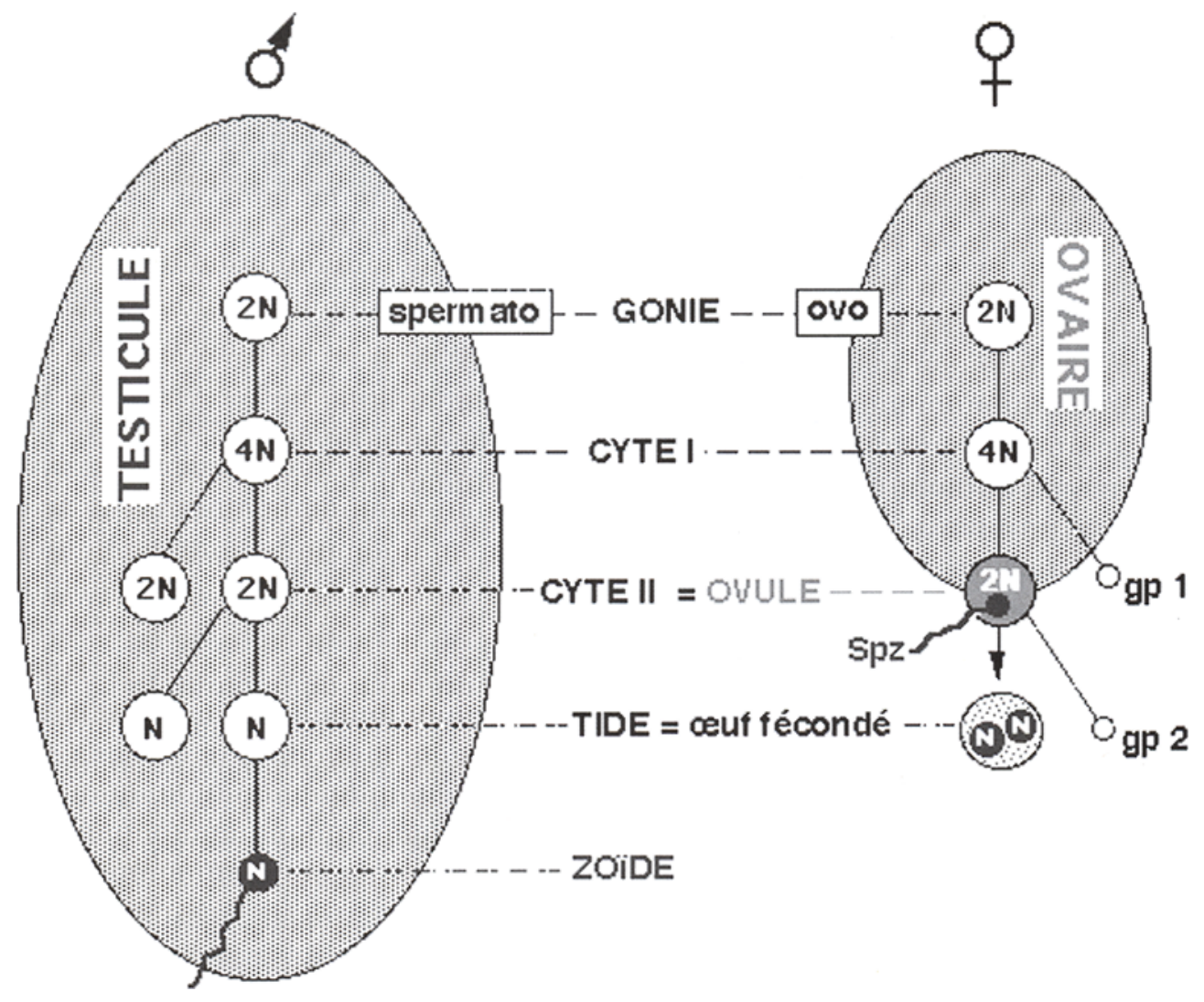

Figure 1 : Comparaison des gamétogenèses mâle et femelle : le gamète femelle "mûr" (ovule) est en fait l'ovocyte II, stade méiotique moins avancé que la spermatide chez le mâle. 
L'arrêt de maturation des spermatides a été récemment constaté dans $7 \%$ des biopsies [35], soit 5 fois moins que dans la série de Chen et al [6]. Ces résultats conflictuels recouvrent certainement des étiologies variées et des techniques d'analyse du testicule plus ou moins performantes. Dans l'hypothèse où l'ICSI serait réalisée avec des spermatides éjaculées [33] on doit évaluer la proportion des hommes montrant une azoospermie non obstructive qui bénéficieraient de cette technique. Nous avons ainsi réalisé une recherche approfondie sur culot cellulaire pour détecter des cellules germinales dans l'éjaculat de 117 hommes azoospermiques. Aucune cellule de la lignée germinale n'a été identifiée chez 22 hommes $(19 \%)$ tandis que des spermatides étaient présentes 89 fois $(76 \%$ ) et quelques spermatozoïdes 6 fois $(5 \%)$.

Une étude récente [15] indique la découverte de spermatides éjaculées dans des proportions équivalentes (69\% des hommes souffrant d'azoospermie non obstructive) et confirme l'absence de corrélation avec les niveaux de FSH.

Ainsi une estimation grossière, tenant compte de l'azoospermie non obstructive chez environ $10 \%$ des couples stériles, permet d'évaluer l'indication d'ICSI avec spermatides éjaculées pour 5 à $10 \%$ de ces couples. Seuls les résultats à venir de cette technique permettront de définir sa place réelle au sein de l'AMP. Outre l'efficacité, il s'agira de confirmer l'innocuité de la fécondation avec spermatide sur la santé et le développement des enfants. Parmi les motivations subjectives qui seront aussi déterminantes, citons l'attitude des couples stériles quant à l'alternative don de sperme et l'acceptation du risque, réel, de transmission de la stérilité de père en fils.

\section{REFERENCES}

1. Ariel M., Cedar H., Carrey J. : Developmental changes in methylation of spermatogenesis-specific genes include reprogramming in the epididymis. Nature Genetics 1994 : 7, 59-63.
2. BLEFCO, RAPPORT A LA DGS : enquête sur l'ICSI en France, 1994-1996.

3. Bonduelle M., Legein J., Buysse A. et Al. : Prospective follow-up study of $\mathbf{4 2 3}$ children born after ICSI. Hum. Reprod. 1996 : 11, 1558-1564.

4. Blendy J., Kaestner K., Weinbauer G., NieschlaG E., ScHÜTZ G. : Severe impairment of spermatogenesis in mice lacking the CREM gene. Nature, 1996 : $380,162-165$.

5. Burruel V., Yanagimachi R., Whitten W. : Normal mice develop from oocytes injected with spermatozoa with grossly misshapen heads. Biol. Reprod, $1996: 55,709-714$.

6. Chen C.S., Chu S.H., Lai Y.M., Wang M.L., ChaN P.R. : Reconsideration of testicular biopsy and follicle-stimulating hormone measurement in the era of ICSI for non-obstructive azoospermia ? Hum. Reprod. 1996 : 11, 2176-2179.

7. Fishel S., Green S., Bishop M. et AL. : Pregnancy after intracytoplasmic injection of spermatid. Lancet 345,24 Juin 1995, 1641-1642.

8. FIVNAT. : Evolution de l'activité ICSI de 1992 à 1995. Contracept. Fertil. Sex. 1996 : 24, 706-709.

9. Goto K., Kinoshita A., Nakanishi Y., OGawa K. : Blastocyst formation following intracytoplasmic injection of in-vitro derived spermatids into bovine oocytes. Hum. Reprod. 1996 : 11, 824-829.

10. KimURA Y, YANAGIMACHI R. : Mouse oocytes injected with testicular spermatozoa or round spermatids can develop into normal offspring. Development 1995 : $121,2397-2405$.

11. KimuRa Y., Yanagimachi R. : Development of normal mice from oocytes injected with secondary spermatocyte nuclei. Biol. Reprod. 1995 : 53, 855862 .

12. Kuretake S., Maleszewsku M., Tokumasu A., FujIMOTO H., YANAGIMACHI R. : Inadequate function of sterile $\mathrm{t}^{\mathrm{w} 5 / \mathrm{t}^{\mathrm{w}} 32}$ spermatozoa overcome by ICSI. Mole Reprod. Dev. 1996 : 44, 230-233.

13. Kuretake S., Kimura Y., Hoshi K., Yanagmach R. : Fertilization and development of mouse oocytes injected with isolated sperm heads. Biol. Reprod. $1996: 55,789-795$.

14. LeE J.D., Kamiguchi Y., Yanagimachi R. : Analysis of chromosome constitution of human spermatozoa with normal and aberrant head morphologies after injection into mouse oocytes. Hum. Reprod. 1996 : 11, 1942-1946.

15. Mendoza C, TESARIK H. : The occurence and identification of round spermatids in the ejaculate of men with nonobstructive azoospermia. Fertil. Steril. $1996: 66,826-829$. 
16. NAGY Z., LIU J., JORIS H. ET AL. : The result of ICSI is not related to any of the three basic sperm parameters. Hum. Reprod. 1996 : 10, 1123-1129.

17. Nantel F., Monaco L., Foulkes N. ET al. : Spermiogenesis deficiency and germ-cell apoptosis in CREM-mutant mice. Nature, 1996 : 380, 159-162.

18. Niss M., VANDERzWalmen P., Vandamme B. ET aL. : Fertilizing ability of immotile spermatozoa after ICSI. Hum. Reprod. 1996 : 11, 2180-2185.

19. OGURA A., YANagimachi R. : Round spermatid nuclei injected into hamster oocytes form pronuclei and participate in syngamy. Biol. Reprod. $1993: 48,219-225$.

20. Ogura A., Matsuda J., Yanagimachi R. : Birth of normal young after electrofusion of mouse oocytes with round spermatids. Proc. Natl. Acad. Sci. USA, 1994 : $91,7460-7462$.

21. Palermo G., Joris H., Devroey P., Van SteirteGHEM A. : Pregnancies after ICSI of single spermatozoa into an oocyte. Lancet $1992: 340,17$.

22. Parrington J., Swann K., Shevchenko V., Sesay A., LAI A. : Calcium oscillations in mammalian eggs triggered by a soluble sperm protein. Nature, 25 Janvier 1996 : 379, 364-368.

23. QUREshI S., Ross A., MA K. ET AL. : PCR screening for Y chromosome microdeletions : a first step towards the diagnosis of genetically-determined spermatogenic failure in men. Mole Hum. Reprod. $1996: 2,775-779$.

24. Rybouchkin A., Dozortsev D., Pelinck M.J., De SutTER P., DHONT M. : Analysis of the oocyte activating capacity and chromosomal complement of round-headed human spermatozoa by their injection into mouse oocytes. Hum. Reprod. 1996, 11, 2170-2175, 1996.

25. SÈle B., Cozzi J., Chevret E., Montell M., RousSEAux S., Pelletier R. : ICSI et syndrome de Klinefelter. Contracept. Fertil. Sex. 1996 : 24, 581-584.

26. Silber S., NAGy Z., LiU J. ET AL. : The use of epididymal and testicular spermatozoa for ICSI : the genetic implications for male infertility. Hum. Reprod. 1995 : 10, 2031-2043.

27. Silber S., Van Steirteghem A., Devroey P. : Sertoli cell only revisited. Hum. Reprod. 1995 : 10, 10311032.

28. Sofikitis N., Miyagawa I., Agapitos E. et al. : Reproductive capacity of the nucleus of the male gamete after completion of meiosis. J. Ass. Reprod. Gen. 1994 : 11, 335-341.

29. Sofikitis N., Toda T., Miyagawa I. et al. : Application of ooplasmic round spermatid nuclear injections (ROSNI) for the treatment of azoospermic men in USA during 1995 (Abst, 55 $5^{\text {th }}$ Meeting Amer. Soc. Reprod. Med., Seattle, USA, Oct 1995, p. 589.
30. Sofikitis N., Toda T., Miyagawa I. ET al. : Beneficial effects of electrical stimulation before round spermatid nuclei injections into rabbit occytes on fertilization and subsequent embryonic development. Fertil. Steril. 1996 : 65, 176.

31. Sutovsky P., Hewitson L., Simerly C. eT AL. : ICSI for Rhesus monkey fertilization results in unusual chromatin, cytoskeletal, and membrane events, but eventually leads to pronuclear development and sperm aster assembly. Hum. Reprod. 1996 : 11, 1703-1712.

32. Svalander P., Jakobsson A.H., Forsberg A.S., BENGTSSON A.C., WiKLAND M. : The outcome of ICSI is unrelated to "strict criteria" sperm morphology. Hum. Reprod. 1996 : 11, 1019-1022.

33. Tesarik J., Mendoza C., Testart J. : Viable embryos from injection of round spermatids into oocytes. New Engl. J. Med., 1995 : 525.

34. TEsTarT J. De la spermatide au spermatozoïde : quels changements nécessaires au développement? Contracept. Fertil. Sex. 1996 : 24, 526-533.

35. Tounnaye H. : Correlation between testicular histology and outcome after ICSI using testicular spermatozoa. Hum. Reprod. 1996 : 11, 2567.

36. VANderzwalmen P., Lejeune B., NŸs M. ET AL. : Fertilization of an oocyte micro-inseminated with a spermatid in an IVF programme. Hum. Reprod. 1995, 10, 502-503.

37. Villar A., Eddy E. Pedersen R. : Developmental regulation of genomic imprinting during gametogenesis. Develop. Biol. 1995 : 172, 264-271.

38. Wolf J. Ph., Feneux D., Escalier D. et al. : Pregnancy after subzonal insemination with spermatozoa lacking outer dynein arms. J. Reprod. Fert. 1993 : 97, 487-492.

\section{ABSTRACT \\ Fertilization by spermatid injection into the oocyte}

\section{J. TestaRT}

There was a recent large spreading of intracytoplasmic sperm injection (ICSI) to treat male infertility in most of in vitro fertilization (IVF) laboratories. The recent data confirm the efficacy of ICSI even by using testicular sperm or sperm with grossly abnormal phenotype (round head, absence of motility). 
Moreover it appears that ICSI could pass beyond the last events of spermatogenesis (i.e. spermiogenesis), since normal development follows fertilization with the male gamete, spermatid, recovered just after completion of meiosis. It is obvious that the natural properties of a mature spermatozoon (motility, ADN compaction, oocyte recognition and penetration) are only necessary to reach the site of fertilization (into the female tube) and to pass through the protective enveloppes around the oocyte (cumulus oophorus, zona pellucida, plasma membrane). The current view that spermatids lack genetic maturation comparing to eggs is not valid since eggs are only secondary oocytes at a meiotic stage equivalent to that of secondary spermatocytes. Moreover genetic imprinting occurs before meiosis, and cytoplasmic structures which seem necessary for embryo development are already present in spermatids.
ICSI using spermatid cells is relevant to men suffering non obstructive azoospermia if spermatids are recovered from either the ejaculate or the testicular tubes. Several normal babies were born after injection of round spermatids. Since these spermatogenic cells are present in the ejaculate of most of the patients with non obstructive azoospermia ( $76 \%$ in our lab), one can estimate to $5-10 \%$ the proportion of sterile men potentially concerned by conception with spermatids. However certain of these men may have occasional sperm found with testicular sperm extraction and it is to early to know if such iatrogenic extraction is always preferable to ejaculate spermatid collection.

Key words : spermatid, ICSI, meiosis, fertilization. 